\title{
Staatsschuld ohne Stigma - eine Illusion?
}

\author{
von Hans-Erhard Haverkampf
}

\begin{abstract}
Die direkte Verwaltung der Staatsschuld durch die politisch Handelnden ist mit zwei Defiziten behaftet: Zum einen stellt der Wirkmechanismus des Finanzmarktes Expertenwissen dar, die politischen Entscheider überschauen die Implikationen ihres Tuns zu wenig. Zum zweiten verstärkt dieses kognitive Manko die Gewohnheit der Akteure, angebotene Sachinformationen je nach politischer Lagerzugehörigkeit zu beachten oder zu verdrängen. Gleichzeitig verfügen die Zentralbanken häufig über jenes Maß an Vertrauen, das bei den staatlichen Instanzen verloren ging. Der nachfolgende Beitrag skizziert das Szenario der Schaffung einer „Europäischen Finanzagentur“, die zwar indirekt demokratisch legitimiert, aber der direkten politischen Steuerung durch die Tagespolitik entzogen wäre. Sie würde, im Konzert mit dem Europäischen System der Zentralbanken und einer effektiven europäischen Marktaufsicht, über die Emission von „Eurobonds“ den Finanzrahmen staatlichen Handelns und die Stabilität des Geldsystems sicherstellen, ohne einem einseitigen und fehlgeleiteten Spardiktat zu verfallen.
\end{abstract}

The administration of public debt by political actors is marked by two deficiencies: firstly, any understanding of the operation of the financial markets is expert knowledge; the political decision-makers cannot adequately judge the implications of their actions. Secondly, this cognitive deficit strengthens the habit of political actors to evaluate or ignore professional information and advice based on party affiliation. At the same time, central banks often command a level of trust that has long been lost by political institutions. The following article outlines the scenario of the creation of a "European Finance Agency", which operates under indirect democratic legitimation, but is removed from direct political control. In concert with the European System of Central Banks and an effective European market oversight, it would issue "Eurobonds" to ensure the financial viability of state action as well as the stability of the monetary system without falling prey to a onesided and misguided call for austerity.

\section{Staatsschulden im globalen Mahlstrom}

Zwei Begriffe der Wirtschaftwissenschaften liegen im Wettstreit um die negativste Konnotation: Staatsschulden und Spekulation. Ein klug inszenierter Mix aus beiden, und schon entstehen Romane zur Zeitgeschichte in großer Auflage: Tom Wolfe legt die Giscard-Anleihe in die Hand des Masters of the Universe zwischen Frühstück und Lunch. Über Bande gespielt, generieren Zinsarbitragen Provisionen i.H.v. mehreren hunderttausend Dollar und wird daraus neuer 
Brennstoff für das „Fegefeuer der Eitelkeiten“. Aus heutiger Sicht sind solche Arbitragen allerdings lediglich Brot- und Buttergeschäft, dies gilt auch für Trendfolgemodelle, die ein Sherman McCoy noch zu Fuß bediente und die heute menschlos im Rechner laufen.

Aber etwas muss dran sein an diesen einfach strukturierten Langweilern, den 10jährigen Bundesanleihen zu 3,5 Prozent, dem spanischen Kurzläufer zu 3,9 Prozent, den amerikanischen Covered Bonds, die zu Schwärmen in chinesische Netze schwimmen. Wobei Schwärme Kollektive ohne Zentrum sind. ${ }^{1}$ Sie folgen, egal ob Fisch oder Anleihe, einer ähnlichen Morphologie des Verhaltens: nicht, solange sie ruhen, erst, wenn sie nomadisieren. Das einzelne Individuum zählt im virtuellen Sammeldepot nicht. Erst das Massenverhalten macht sie für den Jäger attraktiv und zu einem Horror für staatliche Finanzagenturen.

Schon solche Einzelfacetten aktuellen staatlichen Schulden-Managements machen den für die Politik ungewohnten Stress deutlich, bei von der Marktgegenseite inszenierten Kampagnen nicht Räuber oder Opfer, sondern Anwalt des Gemeinwohls zu sein. Das folgt aus zwei Defiziten: Zum einen stellt der Wirkmechanismus des Finanzmarktes Expertenwissen dar. Die politischen Entscheider überschauen die Implikationen ihres Tuns zu wenig. Auch verstärkt dieses kognitive Manko die Gewohnheit der Akteure, angebotene Sachinformationen je nach politischer Lagerzugehörigkeit zu beachten oder zu verdrängen. Eine empirische Studie des ZEW mit 157 Bundestagsabgeordneten als Probanden belegt dies eindrucksvoll. ${ }^{2}$ Einmal unterstellt, das kognitiv-ideologische Problem bei den Akteuren bestünde nicht und der Umgang mit schwarm- wie spieltheoretischem Denken wäre kein Vorteilsprivileg der Spekulanten, dann bliebe als zweites Defizit immer noch die Frage unbeantwortet, ob und wie politisch-administrative Einrichtungen auszugestalten sind, die ein erfolgreiches debt management neuerer Art leisten könnten. Sofort entstünde wieder das bekannte Mixtum aus Sach- und Interessenerwägungen. Die idola fori der jeweiligen politischen Lager motivierten zu einer Auseinandersetzung, welches ideologische Feldzeichen vorangehen dürfte. Solche Probleme kennt die andere Seite des Marktes bekanntlich nicht.

1 Horn, E./Gisi, L.M. (Hg): Schwärme - Kollektive ohne Zentrum, Bielefeld, 2009.

2 Heinemann, F./Janeba, E.: Viewing Tax Policy through Party-colored Glasses: What German Politicians Believe; in: German Economic Review, i.E. 
Scheitert damit nicht schon jeder Versuch, der Staatsverschuldung in allen ihren instrumentellen wie institutionellen Aspekten einen Weg aus den politischen Wertungen frei zu kämpfen und sie allein nach Maßstäben volkswirtschaftlicher Zweckmäßigkeit zu beurteilen und einzusetzen, im Denkansatz?

Der folgenden Ausführungen verstehen sich als Versuch, das Thema „Staatsschuld“ unbelastet von den bisher erkennbaren Beurteilungsreflexen neu zu denken. Die Motivation dazu speist sich aus dem, was Werner Abelshauser in dieser Zeitschrift jüngst als „Verwerfungseffekt“ bezeichnet hat. ${ }^{3}$ In nur drei Jahren türmte sich mit dem Finanzmarktstabilisierungsfonds, der bald obligatorischen europäischen Einlagensicherung, den Risiken des Gesetzes zur „Fortentwicklung der Finanzmarktstabilisierung“, dem so genannten „Griechenlandpaket“ und dem „Europäischen Stabilisierungsmechanismus“ ein finanzielles Schreckensszenario auf. Ist der daraus entstehende Reflex, Hals über Kopf ins Rettungsboot des Schuldenabbaus zu springen, tatsächlich der einzig gangbare Weg?

\section{Die instrumentelle Verschränkung von Geld- und Schulden- politik}

Die ökonomische Theorie liefert bis heute keine belastbaren Antworten auf die Frage nach der zulässigen Höhe von Staatsschulden, ihren makroökonomischen Wirkungen, ihrer Finanzierung, ihrer zeitlichen, räumlichen und sozialen Inzidenz. Dies ist der ökonomischen Theorie insoweit nicht anzulasten, als sie methodisch zu den „Feldwissenschaften“ gehört, nicht also den ceteris-paribusVorteil der Naturwissenschaften genießt.

Innerhalb dieser gleichsam epistemologischen Grenzen erstaunt es umso mehr, als in dem benachbarten, mit großen Schnittmengen zur Schuldenpolitik durchdrungenen Feld der Geldpolitik eine ausdifferenzierte, auf generationenübergreifende Erfahrungen gründende Kunstlehre mit großer Wirkungsmacht in den Händen der Zentralbanken besteht. Ihre Effizienz wuchs und schrumpfte mit dem Abstand zur Politik, wie sich paradigmatisch an Person, Ansichten und Handlungsweisen eines Hjalmar Schacht in der Zeit von der Roggenwährung 1923 bis zu seiner Demission 1937 ablesen lässt. Für die Nachkriegszeit bietet die bis zur großen Reform in den 1990er Jahren noch als politikhörig einzustu-

3 Abelshauser, W.: It's not the economy, stupid!, in: Zeitschrift für Staats- und Europawissenschaften, 8/1 (2010), $1 \mathrm{ff}$. 
fende Bank von Japan ein Lehrbeispiel mangelhaften Umgangs mit der Geldmengensteuerung.

Institutionell bildete sich die Politikferne in Deutschland nach dem Muster der unabhängigen Judikatur. Ein Regelwerk, das Bundesbankgesetz, bestimmte die Aufbauorganisation, die Zielvorgabe (Geldwertstabilität und subsidiäre Ziele) und das Prozedere der Berufung des Leitungspersonals. Der Einfluss beschränkte sich also auf die Metaebene der Geldpolitik in der Einsicht, dass politische Akteure weder jene professionelle Kompetenz noch das interessenfreie Ethos bereitstellen, deren es bedarf. Da das Europäische System der Zentralbanken (ESZB), insbesondere mit dem Übergang zur dritten Stufe nach 1992 in wesentlichen Zügen dem Modell der Bundesbank folgt, gilt diese Politikferne auch für die 17 Eurostaaten.

In die bedeutendsten Aktionsfelder des ESZB neben der Zinspolitik, den Spitzenfazilitäten und der Offenmarktpolitik, gehen hauptsächlich öffentliche Schuldtitel als Besicherungsgrundlage ein. In beiden Sparten traditionellen geldpolitischen Handels sind also öffentliche Schuldtitel bis zur Bewertungsrate „A-“ oder „A3“ herunter für die Geschäftspartner des ESZB geldgleich, im zeitlich begrenzten Ausnahmezustand sogar ein B-Standard.

Für denjenigen Bereich der Offenmarktpolitik, der über Tender Zentralbankgeld schafft, entsteht Liquidität in Höhe der vom ESZB versteigerten Tender, ihrer Fristigkeit und Anzahl. Damit steigt auch die Chance der faktischen Perpetuierung eines Zentralbankkredits. Die eigentliche Stellschraube der Geldmengenregulierung läuft also überwiegend durch die (De-)Monetarisierung öffentlicher Schuldtitel ab.

Sie wirken geldpolitisch noch dominanter durch den Einbezug desjenigen Zweiges der Offenmarktpolitik, der sich dem direkten Kauf/Verkauf öffentlicher Schuldtitel widmet (Satzung des ESZB/EZB, Art. 18.1). Diesen Geschäftszweig sah man bislang durch den Art. 125 AUEV als faktisch tabuisiert an, rechtlich war er es aber keinesfalls. ${ }^{4}$ Nach dieser Lesart galt es, den Haftungsausschluss der EU-Staaten untereinander durch den Aufkauf gefährdeter Schuldtitel nicht gleichsam „durch die Hintertür“ zu unterlaufen. Diese Selbstbindung kennt jedoch keine andere Zentralbank auf der Welt und sie hätte sich im Hinblick auf

4 Protokoll über die Satzung des europäischen Systems der Zentralbanken und der europäischen Zentralbank, Anlage zum Vertrag zur Gründung der Europäischen Gemeinschaft vom 29.7.1992, Art. 18.1, erster Spiegelstrich; Konsolidierte Fassung des Vertrags über die Arbeitsweise der Europäischen Union vom 9.5.2008, AUEV, Art.125.Abs.1 
die Attacken gegen den Euro auch als völlig dysfunktional erwiesen, wurde mithin über Nacht aufgegeben. Die Wirkung auf die Märkte war durchschlagend. Nicht das europäische Stabilisierungssystem vom Juni 2010 wirkte beruhigend, sondern der massive Einsatz der Offenmarktpolitik des ESZB. Hier wird erneut die Differenz von Bundesstaat und Staatenbund deutlich. Der alte und ungelöste Unterschied zwischen Schuldtiteln etwa Schleswig-Holsteins zu denen Griechenlands liegt nicht in der fehlenden Belastbarkeit, sondern in der glücklichen Position des benannten Bundeslandes, seine Zinsfüße unter dem bail-outSchirm der Bundesrepublik trocken halten zu dürfen.

Geldtheoretisch ist der Euro auch ohne „Besicherung“ durch dritte Schuldtitel, insbesondere öffentliche, zu generieren. Diese monetarisieren sich durch den Tenderzuschlag, uno actu, befristet in der Hand der Geschäftsbank. Die Besicherung stellt gleichsam als Sekundärmotiv einen Service für die Staatsfinanzen dar. Sie stärkt die Marktmacht der öffentlichen Anleihen. Aufgrund ihres kaum hinterfragten Akzeptanzwertes bei der EZB lohnt diese Besicherung auch dann ihren Kauf, wenn es die Verzinsung nicht anbietet. Diesen Nachteil versuchen die Geschäftsbanken auszugleichen, indem sie bevorzugt solche Anleihen bevorraten, die einen niedrigen, aber gerade noch akzeptierten Ratinglevel bei naturgemäß höherer Verzinsung aufweisen. Dies gilt jedoch nur bis zur Constant Prepayment Rate (CPR), von der ab etwa ein B-Rating handelsbilanzrechtlich zur Wertberichtigung des öffentlichen Schuldtitels zwingt. Aus dieser Sicht mag der Leser selbst beurteilen, warum bisher (Ende Juni 2010) nur zwei der drei marktbeherrschenden Rating-Agenturen griechische Anleihen zu Ramsch herabgestuft haben, obwohl der Sachverhalt sich für alle drei gleich darstellen müsste.

Die Besicherung von Tendern mit Staatsanleihen folgt jedoch auch symbolischen Zielen. Wenn die Zentralbank als Hort der Geldwertstabilität die staatliche Schuld als Mittel der Wertaufbewahrung nahezu risikofrei einschätzt, adelt sie den öffentlichen Schuldner und verpflichtet ihn zugleich, mit dem hochsensiblen Ruf des „Fiatgeldes“ pfleglich umzugehen. Eine expansive Verschuldung jedenfalls bewirkt symbolisch das Gegenteil. Umso mehr gilt dies für den direkten Handel des ESZB mit öffentlichen Schuldtiteln, da hier ohne den komplexen Umweg über das Bankensystem für jedermann ersichtlich ist, wie dem Staat durch Schöpfung von Zentralbankgeld Kaufkraft zuwächst.

Spätestens mit der Bankenkrise 2008 und dem sich anschließenden ,Verschuldungsprung“ der öffentlichen Hände wandte sich das symbolische Rollenspiel zwischen Staat und Zentralbank aber um. Während vorher die Sicherheit der Staatspapiere bei niedriger Verzinsung als selbstverständliche Voraussetzung 
ihrer Markt- und Besicherungsgängigkeit galt und die Zentralbank dieses asset wie gängige Münze nutzen konnte, wächst sie jetzt in die Rolle, durch ihre Instrumente den gefährdeten Status der öffentlichen Schuld zu stabilisieren. Auf der symbolischen Ebene bedient sich also nicht mehr ein finanziell in seinem Ruf gefestigter Staat einer autonomen geldpolitischen Instanz zum Handel mit der Staatsschuld. Es rettet jetzt vielmehr der Diener den Herrn aus den Strudeln des Glaubwürdigkeitsgefälles Eine Rangumkehr der Autorität sichert dem ESZB jenes $\mathrm{Ma}$ an Vertrauen, das bei den staatlichen Instanzen verloren ging.

Zur symbolischen Ebene des Verbundes von Geldschöpfung und der Besicherung dieses Geburtsaktes durch Aktiva der Banken gehört auch, dass eine unbesicherte Schöpfung derart stark vom Denken der politischen Akteure und des breiten, einzelwirtschaftlich geschulten Publikums abwiche, dass dieses Procedere sofort als Inflationsstimulans gedeutet und die eigene wirtschaftliche Disposition prägen würde; die „Flucht in Sachwerte“ ist eine hier passende Metapher. Die Wirkung der Geldpolitik ist im Ergebnis nicht von ihrer Wahrnehmung zu trennen.

Da sie wesentlich von der öffentlichen Schuldenpolitik und ihrem stimulusresponse-Muster zum ESZB hin überblendet ist, ja dessen integrem Ruf gravierend schaden kann, rückt erneut die Frage in den Vordergrund, wie eine rationale europäische Schuldenpolitik institutionell und instrumentell auszusehen hätte.

\section{Institutionelle Asymmetrie von Schulden- und Geldpolitik}

Festzustellen bleibt, dass der demokratische Souverän im Feld der Geldpolitik sich selbst entmündigt hat, weil seine Entscheidungskultur in diesem Bereich durch vier grundsätzliche Mängel geprägt ist. Entscheidungen erfolgen erstens zu langsam, erfolgen zweitens nicht mit dem erforderlichen komplexen Sachverstand, gefährden drittens durch den Eingang partikularer Interessen das bonum commune und neigen schließlich viertens bei Abwägungsentscheidungen (etwa zwischen den Zielgrößen des StabG) dazu, Nullsummen-Situationen in der Realität durch Formelkompromisse in der Politik zu überdecken und politisch belastende, materiell aber geforderte Handlungsstringenz zu meiden. Nur der Gewöhnungseffekt seit Gründung der Bundesbank und das fehlende Wissen Vieler um die Eingriffstiefe der Geldpolitik in ihre Lebenssituation verschleiert, welches hohe $\mathrm{Ma} ß$ an demokratischer Kompetenz faktisch an eine technokratische Instanz abgetreten wurde. Auf der anderen Seite: Wer die Arbeit des ESZB aus der Nähe betrachtet, kann nur bewundern, mit welchem Reichtum an Registern die 
Geldpolitik instrumentiert ist und wie polyphon dieses Instrument täglich bespielt wird.

Im Gegensatz zum Autonomie-Status der Geldpolitik sind Schulden ein Finanzierungsinstrument in der Hand des Parlaments. Der Rückgriff auf sie gehört zu den Dispositionsrechten der Budgethoheit. Die säkulare Kritik an dieser Zuständigkeit schließt an die vier vorgetragenen Argumente an, die für die Geldpolitik den Autonomie-Status begründeten. Populär und im täglichen Vollzug des parlamentarischen Praxis nachweisbar ist auch ein fünftes: Während Geldpolitik von vornherein durch ihre Komplexität abschreckt und Parlamentarier darin keine Aufgabe sehen, diese mit Hoffnung auf schnelle Renditen bearbeiten zu können, verführt staatlicher Kredit schon aufgrund seiner ,schmerzstillenden Wirkung" zu übergroßer Dosierung. Nichts wäre im politischen Alltagsgeschäft peinigender, als - zwischen konfligierende Interessen gezwängt - keinen Ausweg anbieten zu können, der nur Gewinner kennt. Die sich damit verbindende Abhängigkeit von der Verschuldung wächst zunächst im Verborgenen: Die allmähliche Steigerung der Zinslasten und der Relation zwischen Verschuldung und BIP bleibt lange ohne praktische Folgen und mobilisiert als Zahlenwert kaum kritische Aufmerksamkeit, bis sich unvorhergesehene „SchwarmEreignisse" mit den komplexen Folgen der wachsenden Verschuldung auf der Real- und Wahrnehmungsebene mit für alle sichtbaren Verwerfungen verbinden. Die Haushaltshoheit des Parlaments wandelt sich dann in ein blitzartig wirkendes Haushaltsdiktat gegen die Volksvertretung. Die europäische Gegenwart schreibt dazu das Drehbuch.

Die Reaktion darauf ist dann meist die Forderung nach mehr Parlamentsferne im Bereich der Schuldenpolitik. Eine erste Stufe dazu bildete für die Bundesrepublik die Änderung des Grundgesetzes, insbesondere in Art. 109 GG. Die „Schuldenbremse" genannte nationale Restriktion wendet sich von der 3 Prozent/ 60 Prozent Grenze auf der EU-Ebene radikal ab, hin zum Verzicht auf jegliche Netto-Neuverschuldung, dabei verbunden mit der Hoffnung, die Schuldenquote senke sich durch das unvermeidliche Wachsen des nominalen BIP gleichsam von selbst. Vom Ende her betrachtet erlangte die Bundesrepublik - abgesehen von Fritz Schäffers Juliusturm zu Beginn der 1950er Jahre - einen solchen Finanzstatus bisher nur in einem einzigen Jahr: 2000.

Die dem Schweizer Modell in groben Zügen folgende Neuregelung sucht nun sogar, das Unterlaufen dieser Selbstbindung durch Instrumente wie den Stabilitätsrat (Art. 109a), das Kontrollkonto (115) und Auflagen für die Konsolidierungshilfen (143d) auszuschließen. Die Sollbruchstelle der Schuldenbremse liegt 
dabei zum einen in der geforderten Nullsumme erlaubter zusätzlicher Kreditierungen über einen Konjunkturzyklus hinweg; dieses Kunststück gelang bisher nur Ländern wie der Schweiz oder Kanada. Die Exekutive kann nach Maßgabe eines Bundesgesetzes bestimmen, ob eine Abweichung von der normalen wirtschaftlichen Entwicklung vorliegt und wenn ja, wie das Ende des Abschwungs $\mathrm{zu}$ bestimmen sei und damit die Kreditrückführung zwingend zu beginnen habe. Entscheidungen dieser Art sind aber nicht nur beträchtlichen Abwehrversuchen auf nationaler Ebene ausgesetzt, sondern haben sich einer Phalanx internationaler Einwände aus dem Euro- und EU-Raum, aber auch imperialen Interessenlagen, etwa der USA, zu erwehren. Wie zu verfahren sei, wenn sich eine vom Bundestag festgestellte außergewöhnliche Notlage (Art. 115) mit einem Konjunkturtief zeitlich überschneidet, was in der Tat die Regel sein dürfte, bleibt offen. Jedenfalls sieht das geänderte Grundgesetz für seine Forderungen an keiner Stelle Sanktionen vor, mit Ausnahme von Ländern in Erwartung weiterer Konsolidierungshilfen. Zudem lassen sich globale Entwicklungsszenarien denken, die etwa mit dem Begriff der säkularen Stagnation umrissen werden können. Wenn etwa der Dollar aufgrund der jahrzehntelangen Leistungsbilanzdefizite sein Leitwährungsmonopol abtreten muss, würde er auf einen Tiefstand sinken, der im Ergebnis zu einer Reindustrialisierung der USA auf breiter Front führen dürfte. Das Resultat wäre für die bisherigen „Hauptlieferanten“ der Vereinigten Staaten eine wohl länger anhaltende Überproduktionskrise, noch verstärkt durch eine amerikanische Exportoffensive.

Mit der "Schuldenbremse“ versucht der politische Souverän - aus der Einsicht in die Mängel seiner Tagespolitik - den negativen Staatsschatz „Schulden“ im festen Grund der Verfassung zu verankern. Den juristisch geprägten Teil der deutschen „Angstkultur“ mag das beruhigen, wie ohnehin die stete Aufblähung des Grundgesetzes im Glauben an seine magischen Kräfte begründet sein dürfte. $\mathrm{Ob}$ das die internationalen Interaktionsmuster beeinflusst, in die Deutschland eingebunden bleibt, ist allerdings mehr als fraglich. Der Sturz benachbarter Dominosteine könnte durchaus ausreichen, den eigenen ,,̈̈ber den Kippwinkel“ zu drücken.

Im Folgenden wird deshalb ein wesentlich radikalerer institutioneller Weg zu einer koordinierten europäischen Schuldenpolitik vorgestellt. Sein Vorteil bestünde, ergänzt um eine neue instrumentelle tool box, darin, jährliche Auseinandersetzungen um Verschuldungsquoten samt ihrer Sanktionierung sowohl national als auch supranational überflüssig zu machen. Die absolute oder relative Schuldenhöhe wird dabei als Beurteilungsmaßstab irrelevant. Ähnlich wie im 
Bereich der Geldpolitik bliebe der politische Einfluss auf der Metaebene erhalten, ist ihm aber auf der operativen Ebene genommen. Als Vorbild dient das institutionelle Gerüst der Geldpolitik und die dem verbundene instrumentelle Verschränkung.

Damit zieht diese Analyse auch andere Konsequenzen aus der gegenwärtigen Krise der währungspolitischen Integration Europas als Werner Abelshauser. Er sieht den Ausweg im Rückzug der Geld- und Währungspolitik auf die nationale Ebene, zumindest präferiert er den Weg zurück zur Stufe 2 der Währungsunion. Es würde den Rahmen dieser Ausführungen sprengen, im Einzelnen darzulegen, warum dies so nicht möglich ist. Andererseits dürfte aber auch der gegenwärtige instabile Zwischenzustand nicht haltbar sein, wie bereits ein vielleicht etwas exotischer Ausflug in die Thermodynamik deutlich macht: Eine Währungsunion stellt tendenziell ein gegenüber der Außenwelt in geldpolitischen, währungspolitischen und finanzpolitischen Fragen geschlossenes adiabates System dar. Unter diesen Voraussetzungen strebt das System, einer Thermoskanne vergleichbar, zwangsläufig zur inneren homogenen Ordnung seiner Elemente und damit zum Systemgleichgewicht.

Da eine gemeinsame Schuldenpolitik als Teil der gemeinsamen Geldpolitik der Eurozone unvermeidlich ist, um unzulässige Transfers zwischen den ausgabefreudigen und eher sparsamen Staatshaushalten zu vermeiden, stellt sich sofort die Frage nach den Agenturen, die diese Schuldenpolitik betreiben. Es handelt sich dabei um einen Regelungsbedarf im Binnenbereich der Eurostaaten. Das ESZB hat in den 10 Jahren seines Bestehens zweifellos hervorragende Arbeit geleistet; keine europäische Notenbank vor ihm schaffte es, das 2-ProzentKriterium der Geldwertstabilität durchgängig zu erfüllen. Mit Bezug auf die Meisterung der Griechenland-Frage gilt für das ESZB das gleiche Lob wie es Churchill seinen Piloten im Zweiten Weltkrieg aussprach: „Noch nie haben so viele so wenigen so viel zu danken gehabt." Hätte statt Jean Claude Trichet Axel Weber als Gralshüter der reinen Bundesbanklehre das Zepter in der Griechenland-Krise geführt und keine Marktinterventionen zugelassen, so wäre zumindest der Brüsseler Deckungsstock i. H. v. 60 Milliarden Euro des Europäischen Stabilisierungsfonds schon zur Marktgegenseite gewandert.

Unter der Prämisse, dass die Schuldenpolitik der EU-Staaten nicht mehr zur freien Disposition nationaler Politik und ihrer Einrichtungen steht, liegt es nahe, die dafür zuständige „Agentur“ als eine Art Auftragsverwaltung, jedoch mit eigenständiger Dispositionskompetenz, in das ESZB zu integrieren. Für die folgenden Gedankengänge ist dabei deutlich zwischen den Funktionen einer sol- 
chen „Europäischen Finanzagentur“ (EFA, als Namensleihe der deutschen Einrichtung) im Außenverhältnis zum Finanzmarkt hin und in ihrer Steuerungs-, Abrechnungs- und Servicefunktion nach innen zu ihren 17 Mitgliedern hin zu unterscheiden.

\section{Zuständigkeiten einer Europäischen Finanzagentur (EFA) nach außen}

Die 17 Mitgliedstaaten einschließlich ihrer nachgeordneten Körperschaften emittieren in diesem Konzept ihre Titel gemeinsam und ausschließlich als Eurobonds über den Offenmarkt-Mechanismus des ESZB im Auftrage der Europäischen Finanzagentur, also nicht radizierbar auf einzelne Länder. Höhe und Modus der Verteilung der Erlöse nach innen sind Gegenstand des folgenden Teilkapitels. Diese Agentur existiert bereits in statu nascendi als $\mathrm{GmbH}$ nach luxemburgischem Recht unter dem Dach der Deutschen Finanzagentur in Frankfurt ${ }^{5}$ und soll die Gelder des Europäischen Stabilisierungsmechanismus, wenn notwendig, einsetzen. Da das ESZB als zweitgrößtes Zentralbanksystem der Welt nicht gezwungen ist, unter Zeitdruck und in ungünstige Verhältnisse hinein zu emittieren und marktgerecht dimensionierte Tranchen an Bonds im Auftrag der EFA mit dem Ziel des günstigsten Zinsangebots (analog zum Tendergeschäft) versteigert, ist es unwahrscheinlich, dass im Ergebnis ein wesentlich höherer Zinssatz zum Tragen kommt als aktuell für die Bundesrepublik. Mit Blick auf die Anleihen der deutschen Länder, die finanziell schwächeln, wäre vergleichend mit anfänglich allenfalls 0.3 Prozent höheren als den jeweiligen Bundeszinsen auch für Eurobonds zu rechnen. Allein schon die Existenz des Euro selbst führte nach $2000 \mathrm{zu}$ einer erheblichen Konvergenz der Zinssätze nach unten.

Finanzielle Gravitation entsteht auch durch den relativen Weltanteil und die absolute Größe des repräsentierten BIP. Die EU vereinigte 2008 von den 60,444 Billionen US-Dollar des Bruttoweltprodukts etwa 30,13 Prozent auf sich, gefolgt von der NAFTA mit 28,13 Prozent, Japans mit 8,5 Prozent, der VR China mit 7,19 Prozent und der Russischen Föderation mit 2,8 Prozent. ${ }^{6}$ Es bleibt aus dieser Perspektive unverständlich, warum sich die EU und in ihr die Euro-Zone das Gesetz des Handelns ständig von dritter Seite aus der Hand nehmen lässt.

5 Vgl. Frankfurter Allgemeine Zeitung vom 08.06.2010: „Die Euro-Länder wollen Defizit-Sünder früher bestrafen“; Leiter der Einrichtung ist der Deutsche Klaus Regeling.

$6 \operatorname{UNCTAD}$ (Hg.): Handbook of Statistics, 2009, Section 8.1 (Total of GNP). 
Da das ESZB im Gegensatz zu den Geschäftsbanken einen offenen Rechnungskreis bildet, kann es mit seinem debt management je nach Zinsgunst und Marktlage kurzfristige und langfristige Titel mischen. Es ist an das Fristigkeitsgebot von Aktiva und Passiva bei Geschäftsbanken nicht gebunden. Auch stellt die Höhe des Eigenkapitals kein Limit für die Höhe zeitweise bevorrateter Eurobonds dar, etwa unter der Maßgabe, dass deren eventuelle Wertberichtigung das Eigenkapital aufzehrte und das ESZB so insolvent werden könnte, wie HansWerner Sinn et al. behaupten. ${ }^{7}$ Eigenkapital des ESZB stellt lediglich die finanziell unterlegte Anteilsrate der sie tragenden Mitgliedstaaten dar und damit den Maßstab ihrer Gewinn-/Verlust-Repartierung.

Die Außenvorteile des kollektiven Eurobond-Systems liegen auf der Hand. Es wird wesentlich billiger am Finanzmarkt ,eingekauft“. Dies gilt sowohl für den sich durch Fälligkeiten erneuernden Altbestand an Schulden als auch für eine Nettoneuverschuldung. Man macht nicht mehr ,die Falschen“ reich. Ein weiterer kollektiver Vorteil der Titelfigur „Eurobonds“ liegt in ihrer gleichsam markenbildenden Funktion für den Emittenten EFA (mit ESZB). Als zwar renditearmes, aber standardisiertes sicheres Schuldpapier wird es einen essentiell höheren Grad der Marktdurchdringung erreichen als der bunte Strauß der europäischen Anleihen bisher. Zu bewegen sind also wesentlich größere Volumina. Die Aktionen des ESZB auf dem Weltfinanzmarkt mutierten so zum Bestimmungsfaktor dieser Märkte. Der Zwang zu Anpassung an Gegebenheiten nimmt ab, die Geldpolitik des ESZB wird noch effektiver.

Wer gegen eine einheitlich auftretende Gemeinschaft in Gestalt des EFA/ESZB auf baisse spekulieren will, wird schon an der puren Größe des Systems und seinem im EFA/ESZB inkorporierten Zuwachs an Handlungsintelligenz scheitern. Im Ansatz hat sich dies schon mit der Etablierung des Europäischen Stabilisierungs-Systems gezeigt. Nicht der Fonds selbst mit seinen insgesamt 750 Milliarden Euro gab den Ausschlag, sondern die Anleihen-bezogene Offenmarktpolitik des ESZB. Ohne sie wäre der Euro wahrscheinlich ins Rutschen gekommen und er hätte sich mit enormen Aufkaufverlusten im Fall schlecht bewerteter Anleihen wahrscheinlich erst wieder auf Ebene der Dollar-Parität gefangen.

7 Sinn, H.-W. et al.: Zur Rettung des Euro, in: Frankfurter Allgemeine Zeitung, 18.06.2010. 
Hierzu ein kurzer Exkurs zur Schwarmtheorie am Beispiel einer öffentlichen politischen Demonstration. ${ }^{8}$ Solange sie unter einheitlicher Leitung und Kommunikation mit den Teilnehmern agiert, gilt sie typologisch als zentrierter Schwarm. Löst sich die Rückbindung zur Leitungsebene, etwa aufgrund von Gewaltturbulenzen auf, nomadisiert der Schwarm. Diese „basieren (...) auf der kontinuierlichen Modifikation von Handlungen und Bewegungen durch affektive Signale lokaler Zustände (eigener Zustand, Zustand des nächsten Nachbars, Umweltzustand)“.9 Solche sozialen Körper ohne Zentrum wurden zunächst an Fischen untersucht. William D. Hamilton entdeckte in den 1970er Jahren, warum nomadisierende Schwärme die Tendenz haben, sich räumlich zu verdichten ${ }^{10}$ und fand in dem Frosch-Schlange-Modell die spieltheoretische Lösung dazu. Für das Schwarmindividuum bietet die Dichte des Schwarms eine Verringerung der Wahrscheinlichkeit, Opfer zu werden. Evolutionär bot dieses Verhalten sicher ein Plus an Schutz gegen einzeln auftretende Räuber bei direktem körperlichem Angriff. Bei fern wirkendem Angriff verkehrt sich diese Instinkt-Logik in den gegenteiligen Effekt, wie der makabre „Erfolg“ Buffalo Bills durch wahlloses Schießen vom fahrenden Zug aus in die Herdenmitte von Bisons zeigt. Massenkommunikation entfaltet ebenfalls Fernwirkung: Sie trifft in den Personen, die Anlagevermögen managen und Politikern, die diese Vermögen als Schulden nutzen, auf komplementär symmetrisch wirkende Stimulus-Response Muster des einfachen Zuschnitts „,rette sich, wer kann“ (Anleger) und „stabilisiere, egal wie und mit welchen Kosten“ (Politiker). Die Rettung der beiden amerikanischen Hypothekeninstitute Fanni und Freddie mit einem Gesamtschaden von fast einer Billion US-Dollar bildet sich als Schwarm-Desaster ebenso $a b^{11}$ wie die Schwarm-Logik von automatisierten Trendfolgemodellen im Hochgeschwindigkeitshandel. Der Börsen-Crash an der Wall Street Mitte Mai findet darin ihre Erklärung.

\section{Zuständigkeiten einer Europäischen Finanzagentur nach innen}

Die einzige Prüfkondition der EFA nach innen ist Transparenz der Mitgliedstaaten im Hinblick auf ihr Haushaltsgebaren. Die durchgehend höhere einzelstaatli-

8 Vehlken, S.: Fish \& Chips. Schwärme - Simulation - Selbstoptimierung, in: Horn, E./Gisi, L.M., a.a.O., $125 \mathrm{ff}$.

9 Thacker, E.: Netzwerke, Schwärme, Multitudes, in: Horn, E./Gisi, L.M., a.a.O., 65.

10 Vehlken, S., a.a.O., 165.

11 Vgl. Frankfurter Allgemeine Zeitung vom 15.06.2010: „Billionen-Rettung in Amerika“. 
che Kompetenz von Eurostat wäre Voraussetzung der Teilnahme an der Eurobondsfinanzierung. Eine solche Forderung fände sogar ihre rechtliche Basis in Art. 136 AUEV. Verdeckte Defizitfinanzierungen, wie im Falle Griechenlands durch die Goldman-Sachs-Swaps, aufgeblähte Kassenkredite oder parastaatliche „Metallurgische Forschungsgesellschaften“ seeligen Angedenkens wären schnell aufgeklärt und würden dadurch geahndet, dass der betreffende Staat in der nächsten Runde zu 100 Prozent plus X des gemogelten Betrages von der EurobondsZiehung ausgeschlossen wäre. Es bestünde jedoch kaum ein Anreiz auszuscheren, da die Drittfinanzierung durch wesentlich höhere Zinsen als für Eurobonds abschrecken würde.

Überdies entfallen alle bisherigen wirkungslosen, gleichwohl streitbefangenen Strafverfahren. Die EFA schüttet die als volkswirtschaftlich sinnvoll erachtete kollektive Neu- und Umschuldungssumme nach einem weiter unten zu beschreibenden Verfahren aus. Mit der vorgeschlagenen Etablierung von Eurobonds als dem alleinigen kreditären Kompensat zur Deckung von Budget-Unterschüssen, die durch eine Europäische Finanzagentur bei der ESZB begeben werden, vollzöge sich ein weiterer, diesmal echter Schritt, öffentliche Schulden hinsichtlich ihrer Politiknähe bzw. -ferne auf die gleiche Höhe wie die Geldpolitik zu stellen. Welcher Maßstab durch wen für die Zuteilung dieser Finanzierungskontingente an die Mitglieder des ESZB auch immer gälte, die Budgethoheit der nationalen Parlamente setzte erst nach Verteilung dieser Finanzmittel ein, gerade so, wie bei einer Zuweisung aus „EU-Töpfen“. Der einzelne Mitgliedstaat mutiert so zu einem teilweise geschlossenen Rechnungskreis insofern, als er „nur“ noch sein Findungsrecht für lastenfreie Einnahmen, sprich Steuern, Gebühren, Beiträge etc., behielte. Überschreitet ein Budget-Unterschuss die zugewiesenen Mittel aus Eurobonds-Verkäufen und sind Reserven nicht verfügbar, so müssen die fehlenden Mittel im Umlageverfahren, etwa durch temporäre Steuererhöhungen, eingeworben werden.

An die Stelle der derzeit 17 Einzelparlamente und zugeordneten Exekutiven träfen Organe diese Entscheidungen, wie sie heute bereits für die Geldpolitik existieren. Sie sollten sich möglichst widerspruchsfrei in die bereits bestehenden Einrichtungen integrieren, die für das ESZB mit dem Zentralbankrat als seiner „Legislative“ und auf der eher exekutiven Ebene mit dem Direktorium bestehen. Es wäre auch sachlich geboten, die Entscheidungsgremien der EFA in die Kompetenznähe des EZB-Kerns im ESZB-System zu rücken. Ein „EFA-Rat“ träfe die leitenden Dispositionen, darin dem Zentralbankrat vergleichbar. In ihm sind die berufenen Akteure aus den Mitgliedstaaten versammelt. Überkreuz-Mitglied- 
schaften dürften sich durchaus als sinnvoll erweisen. Wie diese Berufungen nach welchem Schlüssel und sonstigem Prozedere zu erfolgen hätten, wäre Aufgabe einer höheren politischen Arithmetik und ist nicht Gegenstand dieser Darlegungen. Wichtig ist, dass dieser politische Prozess auf der Meta-Ebene Legitimation schafft.

Auf der exekutiven Ebene sollte sich ebenfalls ein dem ESZB analoges „Direktorium“ der EFA bilden, das sich zu guten Teilen mit dem des ESZB personell überschnitte. Diesem wäre zur fachlichen Ergänzung ein Arbeitsstab zuzuordnen, der zum einen die Aufgabe einer ESZB-Rating-Agentur auf der Basis des ECAFRahmenwerkes übernähme. Die jetzigen drei privaten Agenturen mit ihrer eindeutigen Interessenausrichtung verlören damit die Herrschaft über die europäischen Finanzmärkte. Zum anderen versammelte sich der volkswirtschaftliche Sachverstand in der Arbeitsgruppe, womit sich eine sachlich fundierte Empfehlung zur Höhe der Einwerbung von Eurobonds am Markt pro jährlicher Kampagne verbände. Dieser Arbeitsstab hätte eine Hierarchiestufe, die etwa der des „Arbeitskreises Steuerschätzung“ in der BRD entspräche. Sinnvoll wäre es ggf. auch, die dritte Säule des Systems, die Aufsicht über das europäische Bankensystem, wie in Großbritannien beim ESZB anzusiedeln.

\section{Quantitative Auswirkungen des Eurobond-Systems auf die Einzelhaushalte der Mitgliedstaaten}

Im Rahmen eines vom Verfasser entwickelten Simulationsmodells auf der Basis von Grundwerten des Jahres 2009 werden im Folgenden die Auswirkungen des Eurobond-Systems modelltheoretisch untersucht (vgl. Tab. 1).

Tabelle 1: Auswirkungen des Eurobond-Systems

\begin{tabular}{|c|c|c|c|c|c|c|c|c|c|c|c|c|c|c|c|}
\hline & \multicolumn{3}{|c|}{2009 Mrd. $€$ absolut } & \multicolumn{3}{|c|}{\begin{tabular}{|l|} 
Wachstum BIP \\
$\%$ in \\
Alternativen $1-3$
\end{tabular}} & \multicolumn{3}{|c|}{\begin{tabular}{|l|} 
Wachstum \\
Einnahmen \% in \\
Alternativen 1-3
\end{tabular}} & \multicolumn{3}{|c|}{$\begin{array}{l}\text { Wachstum BIP \% } \\
\text { in Alternativen } \\
\text { Schulden 1-3 }\end{array}$} & \multicolumn{3}{|c|}{$\begin{array}{l}\text { Zinsfuß \% in } \\
\text { Alternativen 1-3 }\end{array}$} \\
\hline & BIP & $\begin{array}{l}\text { Steuerauf- } \\
\text { kommen }\end{array}$ & Schulden & 1 & 2 & 3 & 1 & 2 & 3 & 1 & 2 & 3 & 1 & 2 & 3 \\
\hline Deutschland & 2.409 & 1.10 & & 1,5 & 2 & 2,5 & 2 & 2,5 & 1 & 3 & 3,5 & & 2,8 & 2,8 & 3,2 \\
\hline Griechenland & 238 & 87.6 & 273 & 2,5 & 2,5 & 3,0 & 2,5 & 3,5 & 1,5 & 5 & 6,1 & 2 & 5 & 6 & 3 \\
\hline Eurozone & 8.97 & 7.066 & 3.98 & 2 & 3 & 1 & 2 & 2,0 & 1,5 & 3 & 2 & 4 & 3 & 4,2 & 2,5 \\
\hline & & & & 2,5 & 1,5 & 3,5 & 2,5 & 1,5 & 2,0 & 3,3 & 3,0 & 2,0 & 4 & 3 & 2,2 \\
\hline Groß & & & & 1,75 & 2,2 & 2,5 & 2,5 & 2,0 & 1 & 3,2 & 2 & 4 & 4 & 3 & 2,5 \\
\hline Japan & 3644 & 1.603 & 6.916 & 1 & 2 & 1,5 & 3 & 2,0 & 1,5 & 4,5 & 3,5 & 3 & 1,4 & 2 & 2,5 \\
\hline
\end{tabular}

Quelle: Eigenes Simulationsmodell.

Auf der Basis des Jahres 2009 gehen das BIP, die Einnahmenseite des Budgets, der Schuldenstand sowie die unter unterschiedlichen Voraussetzungen prognostizierten Veränderungsraten dieser Bestandsgrößen und der Zinsfuß in dieses 
Modell ein. Die Eingangsgrößen wachsen als geometrische Reihe, also realistischerweise durch den Einbezug des vorjährigen Zuwachses. Bis 2030 als zeitlichem Horizont können damit die unterschiedlich wachsenden Schuldenstände und ihre immer wieder diskutierten benchmarks abgerufen werden: Wann erreicht der Schuldenstand den Gleichstand mit dem BIP, wann tritt der als gefährlich eingestufte 30-Prozent-Anteil des Schuldendienstes an den Budgeteinnahmen ein, wie wirkt sich eine Verzinsungsreduzierung auf die Verschiebung dieser benchmarks aus oder, eher grundlegend, welcher Liquiditätsgewinn ist zu erzielen? Analoge Aussagen trifft das Modell, wenn die anderen Variablen „Schuldenwachstumsrate“, „BIP-Wachstum“ oder „Wachstum der Einnahmenseite/Reduzierung der Ausgabenseite der Budgets“ verändert werden. Die statusquo-Extrapolationen als Kontrast zu einer schuldenpolitisch gewollten Veränderung beruht dabei auf den einschlägigen Zeitreihen von Eurostat. ${ }^{12}$ Bezogen auf die Euro-Länder insgesamt ergibt sich damit folgende Extrapolation (vgl. Tab. 2): Bei 2 Prozent jährlicher BIP-Steigerung, gleicher Rate für das Wachstum der Einnahmen sowie dem durchschnittlichen Zinsniveau der vergangenen 10 Jahre im Euroraum für Anleihen von 4,2 Prozent bei einer Reduzierung dieses Zinsfußes auf 3 Prozent eine kollektive Ersparnis von 456 Milliarden Euro für die Summe der fünf Jahre 2010 bis 2014.

Tabelle 2: Zinsbelastungen und Einsparpotentiale im Euroraum (Extrapolation)

\begin{tabular}{|r|c|c|c|c|c|l|}
\hline Jahr & 2010 & 2011 & 2012 & 2013 & 2014 & $2010-2014$ \\
\hline Zinsbelastung 4,2\% & 306 & 315 & 324 & 334 & 344 & $=1623$ \\
\hline Zinsbelastung 3,0\% & 218 & 225 & 232 & 239 & 253 & $=1167$ \\
\hline & $\overline{88}$ & $\overline{90}$ & $\overline{92}$ & $\overline{95}$ & $\overline{91}$ & $=456$ \\
\hline
\end{tabular}

Anmerkung: Angaben in Milliarden Euro.

Quelle: Eigenes Simulationsmodell.

In der Langzeitbetrachtung Griechenlands bis 2030 würde sich bei einem Wachstum von 2,5 Prozent und einem gleichen Wachstum der Einnahmen, jedoch bei 5 Prozent Schuldenwachstum und 5 Prozent Zinsen, die Zinszahlungs/Einnahmenquote auf 26 Prozent erhöhen. Damit näherte sich Griechenland in 20 Jahren dem Zeitpunkt, an dem es nicht aufgrund einer zu hohen Schuldenquote insolvent würde, sondern weil es den Kern seiner Kollektivgüterproduktion nicht mehr bezahlen kann. Unterlegt man ein stagnierendes BIP und Einnahmeaufkommen bei 5 Prozent Zinsen und einem Schuldenwachstum von 8 Prozent, ist 
dieser Wert schon 2016 erreicht. Nach Maßgabe des Eurobond-Systems, innerhalb dessen der Zwang zur Reduzierung des Haushaltsunterschusses erhalten bliebe, könnte sogar ein haircut unterbleiben. Die Zinsbelastung der Einnahmen betrüge 2030 „nur“ 10,3 Prozent. Realistisch ist wohl, dass die von der EZB aufgekauften Anleihen mit ca. 40 Milliarden Euro noch einmal um die gleiche Summe aufgestockt und gegenüber Griechenland ein Zinsmoratorium auf unbestimmte Zeit ausgesprochen würde, freilich unter der Maßgabe, den Haushaltsunterschuss langfristig bei 2 Prozent zu halten. Dann könnte Griechenland mit einer Zins/Einnahmenquote von 6,5 Prozent bis 2030 gut wirtschaften. Deutlich wird aus den Simulationsrechnungen aber auch, dass die Euro-Staaten in der Summe keine realistische Chance haben, ihren absoluten Schuldenstand bis 2030 wesentlich zu verringern. Ihre Zins/Einnahmenquote würde jedoch im EurobondSystem in 2030 bei tolerablen 6,5 Prozent liegen.

\section{Ist ein Schuldenlimit wirtschaftstheoretisch endogen ableitbar?}

Mit den drei integrierten Säulen ESZB, EFA und Bankenaufsicht entstünde ein europäisches Monetarsystem von hoher instrumenteller Komplexität und Entscheidungsdichte, dem faktisch die makroökonomische Steuerung des Euroraumes zuwächst, während die realwirtschaftlichen Allokations-, Transfer- und Innovationspolitiken bei den Einzelstaaten bzw. der EU verblieben. Dieses System wäre aus institutioneller Sicht ,schwarmsicher" nach innen und nach außen. Es bleibt allerdings die Frage offen, wie Entscheidungsregeln zur Schuldenpolitik mit denen zur Geldpolitik zusammenzuführen und dabei wirtschaftstheoretisch wie letztlich wohlfahrtsökonomisch zu fundieren sind.

Periodisch müssten in dem hier vorgeschlagenen institutionellen Rahmen die „legislativen“ und „exekutiven“ Gremien von EFA und ESZB über den volkswirtschaftlich akzeptablen Umfang der Eurobonds-Emission für die derzeit 17 Mitglieder der Eurozone entscheiden. Die Verteilung des Erlöses erfolgte dann über einen politisch sanktionierten Verteilungsschlüssel. Das bisher im EURaum vertraglich geltende Doppel-Limit von 60 Prozent des BIP und innerhalb dieser Grenzen einer Nettoneuverschuldung von 3 Prozent des BIP, das seitens der deutschen Finanzminister gleichsam zur Monstranz erklärt wurde, bietet außer den politisch situativen Hintergründen seiner Genese keinen nennenswerten ökonomischen Begründungskontext. Die Zufälligkeit einer grob überschlagenen Durchschnittsverschuldung der EU-Staaten zu Zeiten der MaastrichtVerträge sowie die Annahme, ein als durchschnittlich dauerhaft anzunehmendes Wirtschaftswachstum des BIP in Europa von 5 Prozent rechtfertige eine Netto- 
verschuldung von 3 Prozent, stand Pate. Für die Begleitvermutung, eine solche Grenzsetzung garantiere Geldwertstabilität seitens der Staatsfinanzen und keinen Zuwachs der staatlichen Schuldenquote, gibt es keinen empirischen Befund. Die in den vergangenen zehn Jahren realisierte Geldwertstabilität beruht ausschließlich auf der Politik des ESZB, der Zuwachs an staatlichen Defiziten hat sich weder durch die 60/3-Regel, noch durch die dazu eingeführten schwerfälligen Strafverfahren nach Art. 126 AUEV bremsen lassen.

Im Folgenden werden einige der gängigen Maßstäbe geprüft, die möglicherweise als ökonomische benchmarks der Schuldenakkumulation taugen.

\section{Absolute und relative Höhe des Schuldenstandes, Höhe der Zinsbelastung}

Der nationale Schuldenstand schlägt sich in den Bewegungsgrößen des Haushalts einzig als Fluss der Sollzinsen nieder. Aber bereits hier beginnt das Problem. Wenn, wie im Modellfall der Einführung, von für alle verbindlichen Eurobonds die Zinslast auf breiter Front nicht nur für die Neuverschuldung sinkt, sondern auch durch Umschichtung bestehender Schulden, steigt auch das Volumen der Verpflichtungen, die man sich ggf. „leisten“ zu können glaubt, entsprechend. Dem unkontrollierten Anwachsen der Schulden ist aber durch die limitierte Emission der EFA/ZSZB vorgebeugt. Ob dann die steigenden Schulden privaten Kaufkraftentzug bedeuten, ist vom Vollbeschäftigungsgrad abhängig. Der absolute Schuldenstand spielt dabei keine Rolle. Ins Spiel kommt auch liquides Vermögen des Staates (wie im Falle des nicht konvertiblen Yuan Renmin$b i$, dem Auslandsforderungen der VR China zur Gegenrechnung dienen). Bleiben wir bei China: Die Regierung der VR forciert derzeit ein Infrastrukturprogramm, das auch bei wieder erreichter Vollbeschäftigung Kaufkraft vom privaten in den öffentlichen Sektor überführt, wobei ein moderater Anstieg der Geldentwertung in Kauf genommen wird. Volkswirtschaftlich wird also nicht nur Konjunktur-, sondern auch Strukturpolitik betrieben, um volkswirtschaftliche Engpässe durch die unterdimensionierte Kollektivgüterproduktion auszugleichen. Die Staatsschuld wird rapide steigen, rechtfertigt sich aber durch die augenfällige höhere Grenzproduktivität des Infrastrukturbereichs.

Eine ganz andere Rechtfertigung gilt für die japanische Staatsschuld mit mehr als 200 Prozent des BIP. In den Augen von austerity-Verfechtern befindet sich die japanische Volkswirtschaft damit schon auf dem Weg zur Insolvenz. Da aber das japanische Preisniveau seit der Zentralbankreform in den 1990er Jahren stabil 
und die terms of trade tadelsfrei sind, bleibt als einzige Erklärung, dass die anhaltend exzessiv betriebene Sparleistung des privaten Sektors im laissez-faireFalle zu säkularer Stagnation geführt hätte, mit allen schädlichen Folgen einer galoppierenden Deflation bei verstärkter Blasenbildung am Kapitalmarkt (vgl. die Bankenkrise Anfang des Jahrtausends). Folglich blieb das staatliche Defizit bei ebenfalls stagnierendem Außenbeitrag die einzig mögliche und auch ungefährliche Sicherung der relativen Vollbeschäftigung.

Stellen dann die Staatsfinanzen der USA mit ihrem rasanten Wachstum der absoluten und relativen Höhe der Staatsschuld ein bedrohliches Phänomen dar? Auch hier: eher nein. Solange der Dollar als Leitwährung besteht, kann letztlich über das Federal-Reserve-System Dollar immer mit Dollar bezahlt werden. Die durch die jahrzehntelangen Leistungsbilanzdefizite der USA aufgehäuften Dollaraktiva der Überschussländer halten jeden dieser staatlichen Akteure davor zurück, gegen den Dollar zu spekulieren, da dies einer Selbstenteignung gleichkäme. Es wäre auch ein interessantes Gedanken-Experiment, sich vorzustellen, statt eines Ben Bernanke hätte in der Bankenkrise der oben schon bemühte Axel Weber nach seinen Prinzipien das Fed-System steuern dürfen. Dann wären die USA heute aus Prinzipientreue insolvent. Auch für diesen Fall gilt: Nicht der absolute oder relative Schuldenstand ist entscheidend, sondern die seine Veränderung begleitenden Flussgrößen, im Falle der USA die Entwicklung der Kapitalbilanz, der Geldwert, der Beschäftigungsgrad und leider immer noch unbeachtlich: der Saldo der Leistungsbilanz. Das vorhandene geld- und währungspolitische Instrumentarium reicht offensichtlich aus, diese Größen unabhängig von der Entwicklung des Schuldenstandes zu steuern. Allerdings kann das kollektive Gefühl, der Schuldenstand der USA sei unseriös hoch, dazu führen, ein diffuses Misstrauen zu erzeugen, das sich in komplementär angelegten Produkten des Kapitalmarktes widerspiegelt. Das geldtheoretisch interessanteste Produkt dieser Art sind goldgedeckte Indexfonds wie der SPDR Gold Trust. Er hält inzwischen 1.316 Tonnen des Edelmetalls in einem Sondervermögen. Die Transferkosten der Fonds-Anteile sind aufgrund des passiv geführten Managements extrem gering, sodass sich neben dem Dollar anscheinend eine parallele private Goldstandard-Währung herausbildet, die man „mieten“ kann, wie weiland die Noten der Amsterdamer Gold- und Silberbank im 17. und 18. Jahrhundert.

Volkswirtschaftlich ist also die Aussage, ein Schuldenstand sei zu hoch oder noch zu niedrig oder als Grenzwert gerade noch akzeptabel, nicht begründbar. Gemeint können eigentlich immer nur die durch seine Veränderung ausgelösten Veränderungen bei den Flussgrößen der Wechselwirkung sein, also Binnen- und 
Außenwert der Währung, Vollbeschäftigungsgrad, Wachstumspfad, Anwachsen der Zinslast relativ zum jeweiligen Einnahmenniveau des Staates etc. In den Zahlen der benannten Simulationsrechnung ist etwa der Gleichstand der Schuldenlast Deutschlands mit dem BIP erst im Jahre 2030 fast erreicht (95,6 Prozent). Was aber passiert dann? Bricht in der Eifel ein Vulkan aus? Jedenfalls verharrt das Verhältnis von dann 91,8 Milliarden Euro jährlicher Schuldzinsen zur Einnahmeseite des Haushalts bei 5,5\%. Voraussetzung dazu ist noch nicht einmal das Wirksamwerden der Schuldenbremse.

\section{Geldwertstabilität bei Vollbeschäftigung?}

Geldwertstabilität ist eine notwendige, wenn auch nicht hinreichende Voraussetzung für optimale Allokation. Mit diesem Maßstab würde Geld- und Schuldenpolitik gleichnamig gemacht. Die Kooperativgremien EFA und ESZB bestimmen in diesem Denkmodell für jedes Jahr die Summe der Eurobonds, die für den Euroraum gerade noch nicht stabilitätsgefährdend wäre. Mit dem Feinsteuerungspostulat „gerade noch nicht“ wäre makroökonomisch das Sekundärziel Vollbeschäftigung nahe an das der Geldwertstabilität herangeführt. Das bedarf eines multidimensionalen geldpolitischen Interventionsinstrumentariums rund um den oberen Mengenpunkt der Konjunktur. Die Eurobonds-Emission würde so im konkreten Fall auch nationale Konjunkturprogramme gleichsam automatisch mitfinanzieren.

Den aktuellen Gegebenheiten des Marktes entsprechend platziert das ESZB dann die Eurobonds in Tranchen im Auftrag der EFA. Nach innen versteigert die EFA die Erlöse als Kredit-Tender. Ist der Finanzierungsbedarf der Mitgliedsländer erwartbar höher als die geldwertneutrale Gesamtsumme und gingen somit finanzschwache Länder bei den Versteigerungen teilweise leer aus, so könnte etwa das Versteigerungsvolumen auf zwei Drittel des Gesamtvolumens beschränkt und der Rest nach Höhe des jeweiligen BIP repartiert, aber zum Einkaufszins verteilt werden. Ein weiteres, davon getrenntes Auktionsvolumen beträfe die Umschuldung. Länder, die ihr Verschuldungsvolumen nicht abbauen wollen oder können, aber bei fälligen Rückzahlungen durch die billigeren Eurobonds ersetzen müssen, können das im Maßstab 1:1 durchführen. Geldpolitisch wären solche Aktionen neutral und würden Ländern wie Griechenland helfen, ohne die übrigen Staaten zu Kostenträgern zu machen. Das setzt selbstverständlich den Fortbestand restriktiver Haushaltsführung solcher hilfsbedürftiger Staaten voraus. 
Eine solche Form der Deckung von Haushaltsunterschüssen hat mehrere zusätzliche Vorteile:

- Es wäre in Zukunft nicht mehr notwendig, eigene schuldenfinanzierte Konjunkturprogramme aufzulegen. In der Zumessung des jährlichen EurobondsVolumens durch EFA/ESZB wären wirtschaftliche Ungleichgewichte bereits berücksichtigt.

- Notsituationen besonderer Art können durch Sonderziehungen berücksichtigt werden.

- Die Versteigerung der Eurobonds-Tender nach innen führt wegen des Differenzzinses in der Tendenz zu einer räumlichen Verteilung, die der höchsten Grenzproduktivität entspricht.

- Im Bewusstsein der nationalen Politik vollzieht sich zwangsläufig ein Umdenken. Es wird nicht mehr alles beschlossen, was irgendwie wünschbar ist, wobei für den notwendigen Ausgleich der Unterdeckung dann der Finanzminister sorgt. Nach dem neuen Verfahren steht der Finanzrahmen absehbar fest, da die Ressource Eurobonds gleichsam von „oben“ begrenzt ist. Allenfalls die Steuern können angehoben werden.

- Die Zinszahlungen der Mitgliedstaaten fließen an die EFA und über diese an das ESZB (eine Ausnahme bilden die noch nicht abgelösten Altschulden). Für Rechnung der EFA schreibt das ESZB die Zinsen der Eurobonds deren Inhabern gut. Die EFA verrechnet an ihre Mitglieder. Es entsteht aber durch das Offenmarktgeschäft mit Eurobonds eine Art „Schlagschatz“ beim ESZB. Er besteht aus den Differenzzinsen bei Versteigerung der Tender nach innen und dem bevorrateten Volumen an Eurobonds, die noch nicht auf dem Markt platziert sind, aber schon zinsmäßig mit der EFA verrechnet werden. Diese Summen fließen als Bestandteile des Gewinns des ESZB wiederum an die Anteilseigner. Jeder Anteilseigner, der die Tender- oder Repartierungsangebote nicht voll ausgenutzt hat, profitiert davon als „Belohnung“ besonders.

- Es wäre in Zukunft vermutlich wesentlich einfacher, neue Mitglieder in das System aufzunehmen, zumal sie mit diesem Schritt ihre makroökonomischen Zuständigkeiten einbüßen. So könnte die Lücke zwischen den 27 Mitgliedern der EU und den Mitgliedern der Euro-Staaten wahrscheinlich bis auf das „gallische Dorf“ Großbritannien noch bis 2015 geschlossen werden. 


\section{Fazit}

Stabiler Geldwert und ein ungefährer Gleichschritt des Wachstums von BIP und Staatseinnahmen führen zu einem Realzinsniveau, das um diese Werte pendelt. Wenn es gelingt, ein wie oben skizziertes, gleichsam schwarmfestes europäisches Monetarsystem mit den drei Säulen EFA, ESZB und Bankenaufsicht zu institutionalisieren, werden Etatunterschüsse in Zukunft mit Nominalzinsen zu finanzieren sein, die dem Realzinsniveau in etwa entsprechen. Damit hat die Schuldenfrage ihren emotionalen Gehalt und ihre sachlichen Gefahren verloren. Mephisto wird dem Kaiser in Zukunft keine swaps auf künftig zu hebende Bodenschätze mehr unterschieben können. 